\title{
The Development of a High Resolution Mass Spectrometer: A Reminiscence
}

\author{
Alfred O. Nier \\ Sthool of Physics and Astronomy, University of Minnesota, Minneapolis, Minnesota, USA
}

This article is a review of the events leading to the development of the double-focusing tandem mass spectrometer system, which is sometimes called the Johnson-Nier geometry. An essential feature of the geometry is that it eliminates second-order angular aberration, enhancing the sensitivity, without a loss in resolution. Flexibility in the choice of ion source and collector designs is assured because both are outside of regions of electric and magnetic fields. The vacuum housing permits high-temperature baking, ensuring ultrahigh vacuum conditions. Although introduced initially for the purpose of determining precise atomic masses, the design has found its greatest application in studies of structure of heavy molecules, making use of the high resolution to identify fragments. In many cases the composition of a molecule, or fragment, can be deduced from its exact mass by utilizing the known atomic masses of likely constituent atoms. (J Am Soc Mass Spectrom 1991, 2, 447-452)

B efore 1940 mass spectroscopy was employed primarily for the determination of the masses and relative abundances of isotopes. Developments had proceeded in two general directions. The first of these dealt mainly with the measurement of atomic masses. Aston [1] constructed the first mass spectrograph, as he called it, by analogy to an optical spectrograph. A collimated beam of ions was produced in an electrical discharge in a gas at low pressure and sent through an electrical deflection freld followed by a magnetic field. Because the ions had a large spread in velocities, the electric and magnetic frelds were arranged so that ions of a given mass-tocharge ratio, and having a range of velocities, focused at a point on a focal line where a photographic plate, sensitive to ions, was placed. Distances along the photographic plate were correlated with the mass-tocharge ratio of the ions. When the photographic plate was removed from the vacuum and developed, a mass spectrum was observed, and relatively precise masses could be determined from the position of the traces on the plate. Approximate abundances could be found from the blackening of the traces on the plates. During the 1920s and early 1930s Aston determined isotopic masses and abundances for many elements.

Nuclear physics flourished during the 1930s. The development of cyclotrons and high voltage electrostatic generators made it possible to produce ions of hydrogen and helium that had energies of several

Address reprint requests to Alfred O. Nier, School of Physics and Astronomy, University of Minnesota, 116 Church Street, S.E., Minneapolis, MN 55455 million electron volts, which could induce nuclear transformations. As a result of the Einstein relation establishing the equivalence of mass and energy, there was a growing interest in the precise determination of atomic masses because such masses were a measure of the energy content of the nuclei of the atoms.

Other investigators also entered the mass determination field. Herzog [2] showed that with a suitable combination of electric and magnetic sector fields in tandem, or superimposed, ions having a small spread of both velocity and angle as they left an ion source would be focused to a furst order in a mass spectrum along a curve which, in some cases, could be approximated by a straight line for a limited mass range. Such arrangements became known as double focusing in that they focused ions having a range both of velocities and of divergence angles as they left the ion source. In this early work, Herzog did not consider higher order focusing-that is, aberrations proportional to the square of the angular deviation or velocity, or the product of angular deviation and velocity deviation from the central beam of ions. In Austria, Mattauch and Herzog [3] designed a mass spectrograph that possessed properties superior to those of the Aston instrument. In the United States. Dempster [4] at the University of Chicago and Bainbridge and Jordan [5] at Harvard University devised ingenious instruments also capable of determining precise atomic masses. Although there were a few other participants in the field, the principal publications came from the laboratories of the individuals cited. In all of the instruments, mass spectra were recorded on photographic plates, which, upon removal from the vacuum of the mass spectrograph, were developed and, 
after careful measurement of the location of the traces on the plates the masses were determined.

Meanwhile, the measurement of isotopic abundances was proceeding in a somewhat different direction. Dempster [6] constructed an instrument in which mass separation was accomplished with $180^{\circ}$ magnetic deflection that had only angular focusing properties. The ion currents were determined with current-measuring instruments such as electrometers or electroscopes. Abundances of ions were thus determined directly in real time, and the cumbersome photographic plates, followed by readings with optical comparators, were avoided. Instruments of this general type became known as mass spectrometers. They were sometimes referred to as single focusing because they employed only angle focusing. In his early work, Dempster $[7,8]$ determined isotopic abundances for several elements.

During the 1920s there was a growing interest in the study of molecular structure by bombarding molecular gases with electrons to see which fragment ions were formed and what electron energies were required to produce the fragments. Most of the studies employed mass spectrometers based on the 1918 Dempster design. Reviews of the work in the $1920 \mathrm{~s}$ have been given by Smyth [9] and by Kallman and Rosen [10]. The Dempster-type instrument suffered from the handicap that the electric field used for accelerating the ions overlapped that used for accelerating the electrons. As a result, the mass resolution of the instruments was relatively poor, and electronbombarding energies were uncertain. This type of instrument was dramatically improved by the introduction by Bleakney [11] in 1929 of a magnetically collimated electron beam with ions removed in a direction transverse to the electron beam. The revolution that this produced in all future mass spectrometric measurements has been discussed in a recent review article [12].

A mass spectrometer that employs only a magnetic deflection field for producing a mass spectrum cannot be used for making precise measurement of masses. Ions leaving the source have a small spread in energies, and this limits the resolution. Of more importance is the fact that in making precise mass measurements comparison ions, whose masses are close to those of the ion to be measured, are employed. The comparison ions are usually fragments of hydrocarbon molecules. Fragment ions generally acquire some kinetic energy in the ionization and dissociation process so their total energy, which determines their trajectory in the instrument, will be different than that for nonfragment ions. Consequently, instruments that possess both angle and velocity focusing properties, that is, are double focusing, are a necessity.

By 1940 many atomic masses had been measured by using double-focusing mass spectrographs. Relatively accurate isotope abundance ratios had been determined with mass spectrometers employing $180^{\circ}$ magnetic-deflection mass analyzers. During World War II the measurements of atomic masses came to a halt, as did the determination of isotopic abundances of elements in general. However, the interest in separating ${ }^{235} \mathrm{U}$, as well as ${ }^{2} \mathrm{H}$, for the production of nuclear weapons, initially in what was called the "Uranium Project" in the United States-later named the Manhattan Project-led to the development of mass spectrometric techniques of importance to mass spectrometry in the postwar period [13].

In late fall of 1945 I returned to my faculty position at the University of Minnesota after spending essentially all of the previous five years engaged in designing and developing instruments for the uranium program. Promising graduate students were arriving, and we were all anxious to resume research studies we had left before the war. Nuclear physics continued to be one of the leading, if not the leading, area of physics research, and in the United States support was being provided by the newly created Office of Naval Research and by the Atomic Energy Commission, when the latter was established.

Whereas mass spectrometry was still very much an art rather than a science in 1940, the picture changed drastically during the war years. Vacuum and electronic techniques had matured. In our case a wealth of experience in designing and constructing a variety of mass spectrometers [13] had been gained, and when new designs were considered, the performance could be predicted with some certainty. A logical extension of our work was to attempt to make precise atomic mass measurements with a double-focusing mass spectrometer rather than with a mass spectrograph, as was the custom up to that time. Measurements could then be made in real time, and the inconvenience of photographic plates could be avoided. In addition to simplifying the actual measurements, it would be easy to experiment with tuning of the instrument, a tremendous advantage in arriving at optimum performance.

Ours was not the first effort to develop a doublefocusing mass spectrometer, although it was probably the first serious attempt to make precision atomic mass determinations with such an instrument. In 1933 Bondy and Popper [14] described a double-focusing mass spectrometer based on the calculations of Bartky and Dempster [15]. They employed the radial electric field in a $127^{\circ} 17^{\circ}$ sector of a cylindrical condenser superimposed on a constant magnetic field to produce furst-order direction and velocity focusing. Unfortunately, for reasons that are not clear, the resolution obtained, as witnessed by spectra obtained [16], did not approach that then attainable with $180^{\circ}$ single (angle)-focusing magnetic deflection instruments [17]. In 1935 Taylor [18] described an Aston-type instrument for molecular ionization studies in which the photographic plate was replaced by a collector slit and electrometer vacuum tube detector. As was the case for the Bondy and Popper instrument, the perform- 
ance did not appear to be as good as single-focusing mass spectrometers then in use.

In 1938 Bleakney and Hipple [19] described an instrument in which ions moved in trochoidal trajectories in uniform superimposed electric and magnetic fields that were at right angles to one another. Unlike the previously described instruments, the arrangement theoretically gave perfect focusing for both velocity and angular distributions of ions leaving an ion source. The authors did not attempt to apply the instrument to high resolution studies. In 1957 Voorhies et al. [20] conducted a comparative study of various instruments capable of being used for high mass, high resolution investigations. As part of the study, they constructed a large trochoidal instrument, following the Bleakney and Hipple design. The performance was excellent, but the large magnet required and the cost of construction, together with the disadvantage of having the ion source and collector immersed in the electric and magnetic fields, apparently discouraged further development of the method.

In the mass spectrographs used for making precise mass measurements in the prewar period, a flat photographic plate was employed for detecting simultaneously a spectrum of masses. Compromises in design were usually required to achieve good first-order focusing over a region of the spectrum. In conventional magnetic-deflection mass spectrometers employing only a single ion collector, focusing is necessary at only one point, so it appeared it should be possible to avoid the compromises required in the mass spectrographs. It was hoped that, with a judicious arrangement of electric and magnetic lenses, one could obtain second-order angular focusing for a single point, permitting a larger angular spread of ions leaving the ion source and hence greater sensitivity without a loss of resolution.

In 1948 a graduate student, Edgar G. Johnson, appeared in my laboratory looking for a thesis topic for a master's degree. I suggested that he consider the possibility of designing a double-focusing mass spectrometer with improved focusing characteristics. To keep the problem as simple as possible we considered only cases in which the mean ion beam entered and exited from the sector-shaped electromagnetic lenses in directions perpendicular to the field boundaries. The design was further simplified because, in mass spectrometers such as we used, ions are produced by electron impact in Bleakney-type ion sources. Consequently, the spread in energy of the accelerated ions is low, so the principal second-order aberration term that must be considered is that proportional to the square of the angular deviation of ions from the central beam. In studying the problem, Johnson found that, although a number of special cases of secondorder angular focusing had been considered for pure magnetic lenses, only the case in which the object and image distances were zero had been considered for electric lenses such as sectors of cylindrical capacitors.
In none of the cases of superimposed electric and magnetic field elements or tandem electric and magnetic field elements then considered would the second-order angular aberration in the electric lens equal that in the magnetic lens. Consequently, the aberrations could not be used to cancel each other and achieve complete second-order angular focusing. Johnson proceeded to study the general problem of designing tandem combinations of lens elements that would lead to complete second-order angular focusing. Not surprisingly, he found that when combinations of lenses were considered in which at least one of the elements had magnifications other than unity, the angular aberrations could be made to cancel to achieve second-order angular focusing for the combination.

A detailed analysis of the problem [21] showed that an infinite number of combinations that had complete second-order angular focusing could be found. Many of these had geometries that made it impossible to construct practical instruments. Moreover, for the tasks we had in mind there were further criteria to be taken into account. These included:

1. All quantities had to be real, and all field angles had to be positive.

2. The object and image distances for the electric analyzer should be positive and not include zero. Correction for the fringing field at entrance and exit could then be made by using the calculations of Herzog [22].

3. The final image slit had to be away from the magnetic and electric fields so that it would be easy to use various kinds of detection systems for the ions, for example, electron multipliers.

4. The ion source should be sufficiently removed from both the electric and magnetic analyzing fields so that if an ion source magnet was used for collimating an electron beam in the source, its field would not affect the analyzers.

5. The magnetic field sector had to be reasonably small, $90^{\circ}$ or less, so that the cost of the magnet would not be unduly high.

6. The apparatus should not have unreasonable dimensional tolerances that would lead to exorbitant expense of construction.

7. The fabrication of the vacuum housing of the instrument and its contents were to be such that baking at elevated temperatures would be possible to remove impurities that might interfere with the measurements to be made. This meant that the magnet pole faces could not be part of the vacuum housing, as was common in mass spectrographs. Materials such as brass or soft solder had to be avoided, and wax or greased joints could not be employed.

The detailed analysis showed that, whereas a large number of combinations would fulfill the focusing 
Figure 1. Typical double-focusing system employing electric and magnetic lenses in tandem. The ions leave object point, $\mathrm{O}_{e}$, with a half-angle of divergence of $\alpha_{e}$, forming a crossover at $I_{e}$, which serves as the object, $O_{m}$, for the magnetic lens. If the object and image distances for the two lenses are properly chosen, the secondorder angular aberration in the electric lens (seen as an offset of $I_{e}$ from the axis) will be cancelled by the second-order angular aberration in the magnetic lens. The result is no offset at the final image point, $\mathrm{I}_{\mathrm{m}}$. In the original instrument constructed, $\Phi_{e}=90^{\circ}$ and $\Phi_{m}=60^{\circ} ; r_{e}$ and $r_{m}$ were 18.87 and $15.24 \mathrm{~cm}$, respectively; $l_{\mathrm{oe}}, l_{\mathrm{ie}}$ $\mathrm{l}_{\mathrm{om}}$ and $\mathrm{l}_{\mathrm{im}}$ were $6.61,6.61,34.77$, and $20.73 \mathrm{~cm}$, respectively (see ref 21 ). The magnetic lens satisfies the familiar focusing relation $\alpha+\Phi_{\mathrm{m}}+\beta=180^{\circ}$.

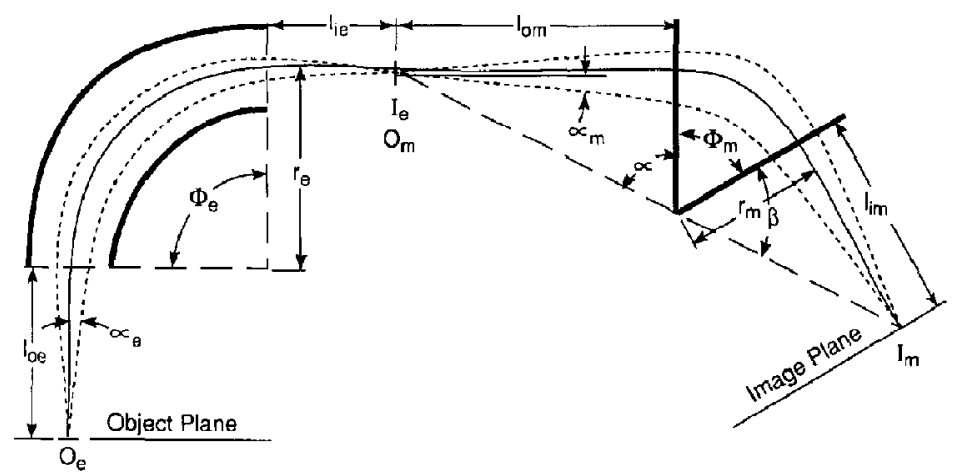

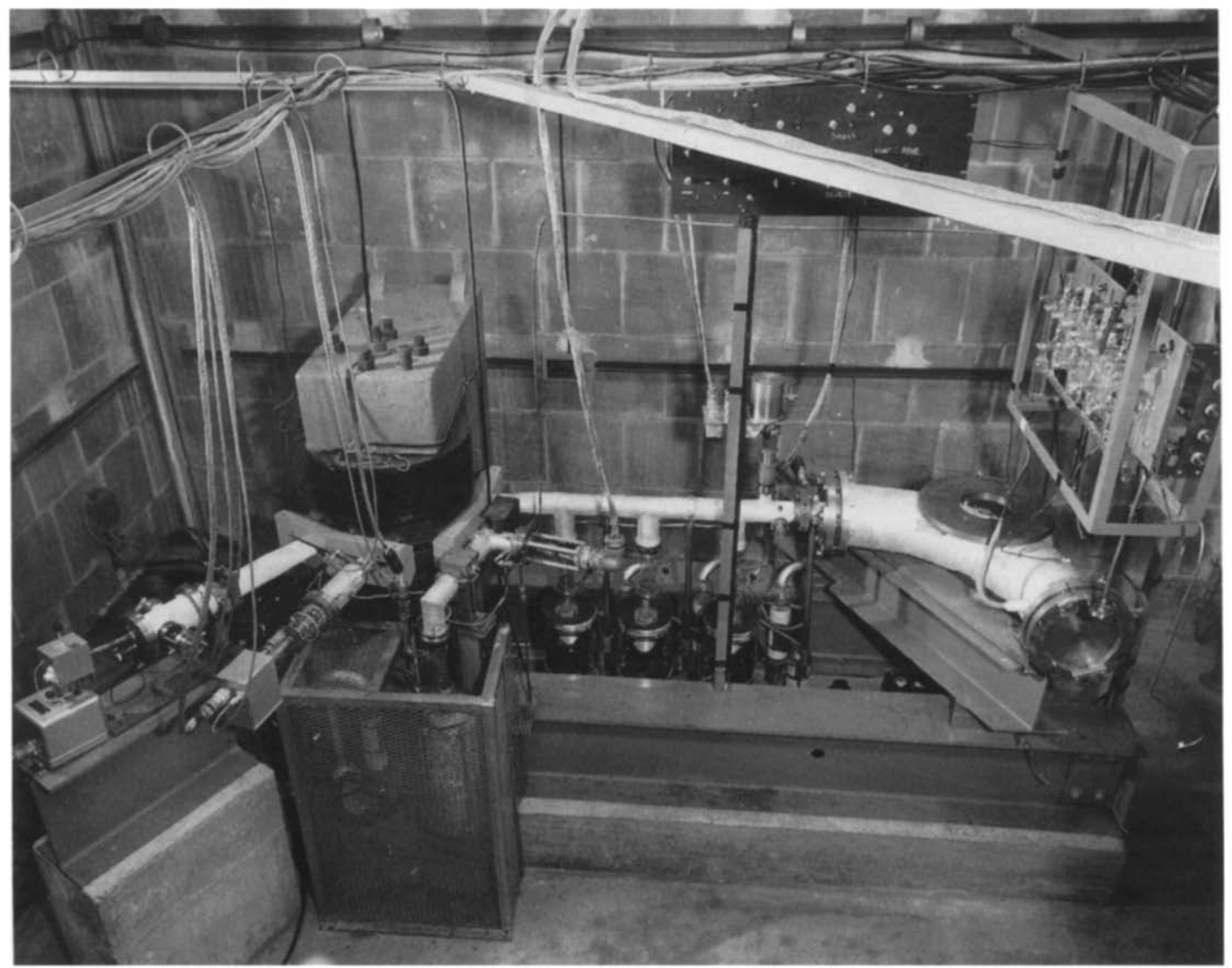

Figure 2. A 1953 photograph of enlarged instrument described in ref 25 , pp 185-193. The vacuum housing is covered with a wrapped heating element insulated with asbestos paper which appears white in the photograph. The instrument is a mirror image of the schematic drawing shown in Figure 1 , so the $90^{\circ}$ electric analyzer appears to the right in the photograph rather than to the left, as in Figure 1. The ion source was not installed at the time the photograph was made. Also seen in the photograph is the auxiliary mass spectrometer used for control purposes [24] in the eariy stages of evolution of the final instrument. 
requirement, only electric sector angles, $\Phi_{\mathrm{e}}$, larger than $65^{\circ}$ would satisfy several of the conditions just cited. The dispersion depends only weakly upon the angle. Accordingly, in the interest of simplicity in construction $\Phi_{e}$ was arbitrarily chosen to be $90^{\circ}$. Likewise, the magnetic-lens sector angle was chosen to be $60^{\circ}$, largely because we had experience in building magnets with this configuration. Other angles, such as $90^{\circ}$, would have served equally well.

Figure 1 is a schematic drawing showing the electric and magnetic sector lenses and the trajectories of a diverging bundle $\left(2 \alpha_{\mathrm{e}}\right)$ leaving a point source on the object plane. In the figure the second-order angular aberration of the electric analyzer appears as the displacement of the image $I_{e}$ from the axis of the instrument. This image then serves as the object $O_{m}$ for the magnetic lens whose angular aberration cancels that of the electric lens. The net result is that the original diverging beam of ions is sharply focused to the second order at $I_{m}$ on the axis of the instrument. The various critical dimensions for the first instrument constructed are listed in the caption to Figure 1 . The radius of curvature of the central beam in the magnetic lens was arbitrarily chosen as $r_{m}=6$ inches $(15.24 \mathrm{~cm})$ inasmuch as we had experience in building single-focusing magnetic instruments of this size. Later, an instrument approximately three times as large was constructed, and with it many of the atomic masses now listed in compilations and tables were determined. A photograph of this instrument is shown in Figure 2.

The precision determination of atomic masses by magnetic-deflection mass spectroscopy requires high stability of the electric and magnetic fields that determine the trajectories of the ions. In mass spectrographs this was accomplished by using batteries of adequate capacities as sources of power. An important reason for making the poles of the magnets part of the vacuum housing of those instruments was that this reduced the air gap and hence the power requirement of the magnets.

In the initial instrument described here, a different approach to stability was employed [23]. An auxiliary small single-focusing mass spectrometer was mounted so that it would experience the same magnetic field as the larger double-focusing instrument. It derived its inn-accelerating potential from the same power supply as the double-focusing instrument and had a split ion collector with a differential amplifier for detecting its ion beam. A variation in either the magnetic field or the power supply providing the ion accelerating and deflection fields for the two instruments would cause an imbalance of the current at the collectors of the single-focusing instrument. The imbalance sent a signal to the power supply providing the ion accelerating and deflection fields, which in turn restored the ion trajectories to their original state. The arrangement, in effect, used the constancy of the mass of the gas in the small spectrometer tube as the reference

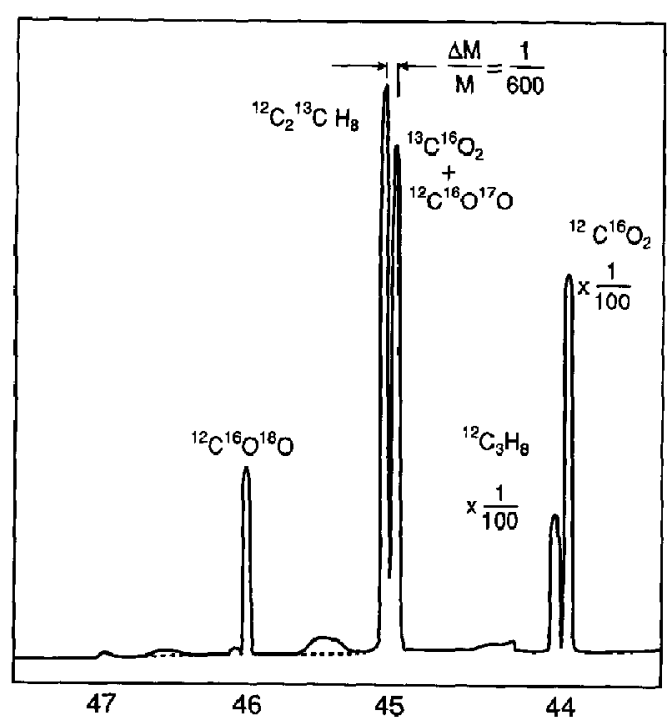

Figure 3. Mass spectrum over the 44-47 mass region when a mixture of $\mathrm{CO}_{2}$ and $\mathrm{C}_{3} \mathrm{H}_{8}$ was introduced into the instrument shown in Figure 1. When this spectrum was ran, the sourcedefining slit that served as $\mathrm{O}_{\mathrm{e}}$ had a width of $0.125 \mathrm{~mm}$, and the final image slit at $I_{m}$ a width of $0.150 \mathrm{~mm}$. The small broad peaks, which fall between masses 46 and 47 and between 46 and 45 , are artifacts due to scattered ions not properly screened out at the time the data were taken.

standard for the system. It, with improvements [24], was employed for a number of years until improved "peak-matching" methods of measurement were adopted.

Because of the interest in nuclear physics and the precise determination of atomic masses, the early 1950s saw the development of a variety of mass spectrometers and spectrographs. Some employed double-focusing, others time-of-flight or ion-resonance techniques. Generally, high resolution was achieved through the combination of ingenious principles with large size. In most cases the instruments were one of a kind. A discussion of them is beyond the scope of this article. The reader is directed to a number of references for details [25-28]

Although the instrument described here was developed initially for the purpose of making precise atomic mass determinations, its resolution, higher than that of then-existing single-focusing mass spectrometers, suggested that it might be useful for making isotope abundance measurements in cases where impurities present might interfere with the measurement of low abundance isotopes or for making gas analyses of mixtures of heavy molecules. In a review paper [29] that discussed the status of isotope abundance and mass determinations in 1954, a spectrum was shown, which appears here as Figure 3 , that illustrated the complete separation of the molecules $\mathrm{CO}_{2}$ and $\mathrm{C}_{3} \mathrm{H}_{8}$. which have the same mass number, 44 , but differing in mass by 1 part in 600 . It was pointed out that 
instrumentation of the type described had the potential for identifying the composition of heavy hydrocarbons from the exact masses of the constituent atoms. This prophesy has indeed come true as witnessed by the large number of high resolution double-focusing mass spectrometers in use in chemical laboratories today. Indeed, in terms of the number of instruments in use and the personnel involved, the applications to problems of molecular structure have far exceeded the research applications for which the instrument was originally developed. A better example of the interdependence of different fields of scientific inquiry can hardly be found.

\section{References}

1. Aston, F. W. Philos. Magn. 1919, 38, 707-714.

2. Herzog, R. Z. Phys. 1934, 89, 447-473.

3. Mattauch, J.; Herzog, R. Z. Phys. 1934, 89, 786-795.

4. Dempster, A. J. Proc. Am. Philos. Soc. 1935, 75, 755-767.

5. Bainbridge, K. T.; Jordan, E. B. Phys. Rev. 1936, 50, 282-296.

6. Dempster, A. J. Phys. Rev. 1918, 11, 316-325.

7. Dempster, A. J. Phys. Rev. 1921, 18, 415-422.

8. Dempster, A. J. Phys. Rev. 1922, 20, 631-638.

9. Smyth, H. D. Rev. Mod. Phys. 1931, 3, 347-391.

10. Kallman, H.; Rosen, B. Phys. Z. 1931, 32, 521-544.

11. Bleakney, W. Phys. Rev. 1929, 34, 157-160.
12. Nier, A. O. Int. I. Mass Spectrom. Ion Processes 1990, 100, 1-13.

13. Nier, A. O. J. Chern. Edtucation 1989, 66, 385-388.

14. Bondy, H.; Popper, K. Ann. Phys. 1933, 17, 425-444.

15. Bartky, W.; Dempster, A. J. Phys. Rev. 1929, 33, 1019-1022.

16. Bondy, H.; Johannsen, G.; Popper, K. Z. Phys. 1935, 95, $46-52$.

17. Nier, A. O. Phys. Rev. 1936, 50, 1041-1045.

18. Taylor. D. D. Phys. Rev. 1935, 47, 666-671.

19. Bleakney, W.; Hipple, J. A., Jr. Phys. Rev. 1938, 53, 521-529.

20. Voorhies, H. G.; Robinson, C. F.; Hall, L. G.; Brubaker, W. M.; Berry, C. E. In Advances in Mass Spectrometry; Waldron, J. D., Ed,; Pergamon: London, 1959; p 44.

21. Johnson, E. G.; Nier, A. O. Phys. Rev. 1953, 91, 10-17.

22. Herzog, R. Z. Phys. 1935, 97, 596-602.

23. Nier, A. O.; Roberts, T. R. Phys. Rev. 1951, 81, 507-510.

24. Nier, A. O. In Mass Spectroscopy in Physics Research; National Bureau of Standards Circular 522; U.S. Government Printing Office: Washington, DC, 1953; p 29.

25. Nuclear Masses and their Determination; Hintenberger, $\mathrm{H}$., Ed.; Pergamon: London, 1957.

26. Duckworth, H. E. Mass Spectrometry; Cambridge University Press: Cambridge, 1958.

27. Mass Spectrometry; McDowell, C. A., Ed.; McGraw-Hill: New York, 1963.

28. White, F. A.; Wood, G. M. Mass Spectrometry-Applications in Science and Engineering; John Wiley: New York, 1986.

29. Nier, A. O. Z. Elektrochem. 1954, 58, 559-567; reprinted in Science, 1955, 121, 73\%-744. 